\title{
Efficacy of coronary sinus reducer implantation in patients with chronic total occlusion of the right coronary artery
}

\author{
Miha Mrak ${ }^{1,2}$, Nejc Pavšič ${ }^{1,2}$, Francesco Ponticelli3,4, Alessandro Beneduce ${ }^{4}$, Anna Palmisano ${ }^{5}$, \\ Stefano Guarracini ${ }^{6}$, Antonio Esposito ${ }^{4}$, Shmuel Banai ${ }^{7}$, David Žižek ${ }^{1,2}$, Francesco Giannini' ${ }^{3}$, Matjaž Bunc ${ }^{1,2}$ \\ 'Department of Cardiology, University Medical Center Ljubljana, Ljubljana, Slovenia \\ ${ }^{2}$ Faculty of Medicine, University of Ljubljana, Ljubljana, Slovenia \\ ${ }^{3}$ Interventional Cardiology Unit, GVM Care and Research Maria Cecilia Hospital, Cotignola, Italy \\ ${ }^{4}$ Interventional Cardiology Unit, IRCCS San Raffaele Hospital, Milan, Italy \\ ${ }^{5}$ Radiology Unit, IRCCS San Raffaele Hospital, Milan, Italy \\ ${ }^{6}$ Department of Cardiology, Pierangeli Clinic, Pescara, Italy \\ ${ }^{7}$ Tel Aviv Sourasky Medical Center, Tel Aviv, Israel
}

\section{Editorial}

by Konigstein

Correspondence to: Prof. Matjaž Bunc, MD, PhD, Department of Cardiology, University Medical Center Ljubljana,

Zaloška 7, 1000 Ljubljana,

Slovenia,

phone: +38615223947 e-mail:mbuncek@yahoo.com

Copyright by the Author(s), 2022

DOI: 10.33963/KP.a2021.0132

Received:

June 7, 2021

Accepted:

October 11, 2021

Early publication date: October 12, 2021

\section{A B S TR A C T}

Background: Clinical efficacy of coronary sinus reducer (CSR) in refractory angina (RA) patients with ischemia due to the chronic total occlusion (CTO) of the right coronary artery (RCA) remains unknown.

Aims: To evaluate the efficacy of CSR implantation in RA patients with CTO RCA and compare them to CSR recipients with left coronary artery (LCA) ischemia.

Methods: Consecutive patients with CTO RCA from 2 centers were prospectively included and compared to patients with LCA ischemia. All patients underwent evaluation of angina severity and quality of life (QoL) at baseline and after 12 months. In a subgroup of CTO RCA patients, stress cardiac magnetic resonance (CMR) imaging was also performed.

Results: Twenty-two patients with CTO RCA and predominant inferior and/or inferoseptal wall ischemia (the CTO RCA group) were compared to 24 patients with predominant anterior, lateral, and/or anteroseptal wall ischemia (the LCA group). While the Canadian Cardiovascular Society (CCS) angina score mean (SD) improved in the CTO RCA group from $2.73(0.46)$ to $1.82(0.73)(P<0.001)$ and in the LCA group from $2.67(0.57)$ to $1.92(0.72)(P<0.001)$, there was no intergroup difference $(P=0.350)$. Significant improvement in all domains of the Seattle Angina Questionnaire was observed. Stress CMR did not show a significant reduction of ischemic inferior and/or inferoseptal segments, however, improvements in the transmurality index $(P=0.03)$ and the myocardial perfusion reserve index in segments with inducible ischemia $(P=0.03)$ were observed in the CTO RCA group.

Conclusions: In CTO RCA patients, CSR implantation alleviated angina symptoms and improved QoL. The extent of improvement was comparable to that observed in patients with LCA ischemia.

Key words: chronic total occlusion, coronary sinus reducer, refractory angina pectoris, quality of life

\section{INTRODUCTION}

Coronary sinus reducer (CSR) implantation is a treatment option for patients with disabling refractory angina (RA). Procedural safety and long-term efficacy of CSR have been demonstrated in patients with obstructive coronary artery disease (CAD), non-obstructive CAD, and microvascular dysfunction [1-6]. However, as CSR is implanted in the coronary sinus distal to the venous drainage of the right coronary artery (RCA) territory (the middle cardiac vein), this treatment is thought to be 
WHAT'S NEW?

Coronary sinus reducer (CSR) implantation is a novel treatment option for patients with angina pectoris refractory to optimal medical therapy. Patients with angina due to obstructive disease of the right coronary artery (RCA) were excluded from previous CSR studies. The results of our study are the first to show that CSR implantation in patients with chronic total occlusion (CTO) of the RCA and predominant inferior and inferoseptal wall ischemia improves angina symptoms and quality of life. The symptomatic improvement is comparable to that observed in patients with obstructive disease of the left coronary artery.

ineffective in patients with predominant ischemia in the inferior and/or inferoseptal left ventricular wall (LVW) [7].

Patients with chronic total occlusion (CTO) of the RCA differ from patients with non-occlusive RCA disease by the presence of a developed collateral network, mostly originating from the left anterior descending (LAD) and circumflex (LCX) coronary arteries [8]. Several studies have shown that coronary venous pressure elevation following coronary sinus obstruction augments collateral flow and enhances local myocardial perfusion by normalizing the ratio between sub-epicardial and sub-endocardial blood flow [9-11]. Therefore, it is reasonable to speculate that symptomatic benefit from CSR implantation could be achieved even in RA patients with predominantly CTO RCA-related ischemia.

This study aimed to evaluate the clinical benefit of the CSR in RA patients with CTO RCA and compare them to patients with angina caused by obstructive CAD of the left coronary artery (LCA).

\section{METHODS}

\section{Study design and patient selection}

Consecutive RA patients with CTO RCA and predominant inferior and/or inferoseptal LVW ischemia from 2 centers were included in this prospective observational study between June 2016 and November 2019. CTO was defined as a complete occlusion of the coronary artery with Thrombolysis in Myocardial Infarction grade 0 (no flow) present for at least 3 months. In the case of concomitant coronary artery bypass grafting, occluded RCA with no patent graft to the distal vessel was determined as CTO RCA.

Inclusion criteria for CSR implantation were age $>18$ years, RA with the Canadian Cardiovascular Society (CCS) angina score 2-4 despite at least 3-months optimal medical therapy at maximally tolerated doses, obstructive CAD without further revascularization options, and objective evidence of reversible ischemia as assessed by single-photon emission computed tomography (SPECT) or stress cardiac magnetic resonance (CMR) imaging. The antianginal therapy was considered optimal if the patient was receiving firstline therapy and at least one second-line antianginal agent (long-acting nitrate, ranolazine, or ivabradine). Patients with acute coronary syndrome in the last 3 months, successful percutaneous coronary intervention, or coronary artery bypass grafting in the last 6 months were excluded.
Patients with CTO RCA and predominant inferior and/or inferoseptal LVW ischemia were compared to a control group of consecutive patients with obstructive CAD and predominant anterior, lateral, and/or anteroseptal LVW ischemia, who received CSR in the same time frame. All patients underwent a baseline and follow-up clinical evaluation after 12 months. Angina severity and quality of life (QoL) were assessed in all patients, while in a subgroup of CTO RCA patients stress CMR imaging was also performed. Medical therapy had to remain unchanged during the follow-up.

The study was conducted in compliance with the provisions of the Declaration of Helsinki. All patients provided informed consent before implantation, and the study was approved by the local ethics committee.

\section{Implantation procedure}

The implantation procedure of the CSR device (NeovasC Reducer, Neovasc Inc., Richmond, Canada) was previously described [7, 12]. All procedures were performed through right jugular venous access. All patients received dual antiplatelet therapy for at least 1 month. Predefined procedural complications included procedural death, myocardial infarction, coronary sinus perforation, cardiac tamponade, and jugular venous access site complications.

\section{Angina severity and quality of life assessment}

Angina severity and QoL were assessed by the CCS score and the Seattle Angina Questionnaire (SAQ). Clinical evaluation and the CCS grading were performed during out-patient visits by physicians not involved in patient selection or implantation procedure, while SAQ was completed by each patient alone. The CCS class was graded on a scale of 1 to 4 depending on the clinical information provided by the patient while the SAQ score was calculated for each of the five domains separately (physical limitation, angina stability, angina frequency, treatment satisfaction, and QoL).

\section{Stress cardiac magnetic resonance imaging protocol}

Stress CMR imaging using a 1.5T CMR scanner (Philips Ingenia, Best, The Netherlands) equipped with a 32-channel body coil was performed before and 4 months after CSR implantation in the subgroup of CTO RCA patients. Pharmacological stress was induced by dipyridamole infusion. Intravenous aminophylline was administered to neutralize the stressor after first-pass perfusion imaging acquisition, 
followed by rest perfusion imaging 10 minutes later. All CMR analyses were performed by 2 experienced readers. Blinding was not possible because CSR is visible on CMR.

For myocardial perfusion analysis the American Heart Association 16-segment left ventricular (LV) model was used with segments 4,10 , and 15 representing the inferior LVW and segments 3 and 9 in the inferoseptal LVW. Several parameters were used for myocardial perfusion analysis. Standard visual analysis of inducible perfusion defects, defined as myocardial areas with visible hypo-enhancement persisting for at least 3 frames after the LV cavity peak enhancement, was done [13]. Transmural extension of inducible ischemia in each myocardial segment was presented as the "transmurality index" - 0: absent ischemia; 1 : ischemia $\leq 50 \%$ wall thickness; 2 : ischemia $>50 \%$ wall thickness. The total myocardial ischemic burden was defined as the percentage of LV mass affected by inducible ischemia divided by the total LV mass. The myocardial perfusion reserve index (MPRI), a novel semiquantitative parameter of myocardial perfusion, was also used. The MPRI may supplement visual analysis and is used in both research and clinical diagnostic work-up of patients with CAD. It was defined as the stress to rest ratio of myocardial signal intensity upslopes normalized to LV input, with higher MPRI values indicating better perfusion reserve (formula [myo- cardial upslope stress/LV cavity upslope stress]/[myocardial upslope rest/LV cavity upslope rest]) [12-14].

\section{Statistical analysis}

Categorical variables are represented as frequencies and percentages and were compared using chi-square and Fisher exact tests as appropriate. Continuous variables are presented as mean (standard deviation [SD]) or as median (interquartile range $[\mathrm{IQR}]$ ). The normality of distribution was tested by the Kolmogorov-Smirnov test. Intra- and intergroup differences were compared with the use of the independent or paired-sample Student t-test, the Wilcoxon rank-sum test, the Wilcoxon signed-rank test, and the McNemar test as appropriate. A two-sided $P$-value of 0.05 was considered statistically significant. Statistical analysis was performed in IBM SPSS Statistics for Windows, version 22.0 (IBM Corp., Armonk, NY, USA).

\section{RESULTS}

\section{Baseline characteristics}

Twenty-two consecutive patients with CTO RCA and predominant inferior and/or inferoseptal LVW ischemia (the CTO RCA group) were compared to 24 patients with obstructive CAD and predominant anterior, later-

Table 1. Baseline characteristics of the study population

\begin{tabular}{|c|c|c|c|c|c|}
\hline & LCA $(n=24)$ & CTO RCA $(n=22)$ & Isolated CTO RCA ( $=12)$ & P-value ${ }^{a}$ & P-value ${ }^{b}$ \\
\hline Age, years, mean (SD) & $73.6(6.6)$ & $71.5(8.2)$ & $70.8(9.2)$ & 0.33 & 0.12 \\
\hline Male sex, n (\%) & $21(87.5)$ & $21(95.5)$ & $11(91.7)$ & 0.61 & 1.0 \\
\hline Hypertension, n (\%) & $21(87.5)$ & $21(95.5)$ & $11(91.7)$ & 0.61 & 1.0 \\
\hline Hyperlipidemia, n (\%) & $23(95.8)$ & $19(86.4)$ & $10(83.3)$ & 0.34 & 0.25 \\
\hline Diabetes mellitus, n (\%) & $11(45.8)$ & $7(31.8)$ & $4(33.3)$ & 0.33 & 0.47 \\
\hline Previous myocardial infarction, n (\%) & $17(70.8)$ & $13(59.1)$ & $7(58.3)$ & 0.41 & 0.48 \\
\hline Previous percutaneous coronary intervention, $\mathrm{n}(\%)$ & $16(66.7)$ & $16(72.7)$ & $9(75)$ & 0.65 & 0.71 \\
\hline Previous coronary bypass graft, n (\%) & $20(83.3)$ & $14(63.6)$ & $4(33.3)$ & 0.13 & $<0.01$ \\
\hline \multicolumn{6}{|l|}{ Ischemia territory } \\
\hline Anterior, n (\%) & $9(37.5)$ & $4(18)$ & 0 & 0.15 & 0.02 \\
\hline Inferior, $\mathrm{n}(\%)$ & $2(8.3)$ & $22(100)$ & $12(100)$ & $<0.01$ & $<0.01$ \\
\hline Lateral, n (\%) & $18(75.0)$ & $3(13.4)$ & 0 & $<0.01$ & $<0.01$ \\
\hline Septal, n (\%) & $5(20.8)$ & $12(54.5)$ & $3(25.0)$ & 0.02 & 1.0 \\
\hline Apical, n (\%) & $5(20.8)$ & 0 & 0 & 0.05 & 0.15 \\
\hline \multicolumn{6}{|l|}{ Rentrop grade } \\
\hline Grade 0, n (\%) & $\mathrm{n} / \mathrm{a}$ & 0 & 0 & & \\
\hline Grade 1, n (\%) & $\mathrm{n} / \mathrm{a}$ & $3(13.6)$ & 0 & & \\
\hline Grade 2, n (\%) & $\mathrm{n} / \mathrm{a}$ & $15(68.2)$ & $8(66.7)$ & & \\
\hline Grade 3, n (\%) & $\mathrm{n} / \mathrm{a}$ & $4(18.2)$ & $4(33.3)$ & & \\
\hline \multicolumn{6}{|l|}{ Medical therapy } \\
\hline Acetylsalicylic acid, n (\%) & $21(87.5)$ & $21(95.5)$ & $11(91.7)$ & 0.61 & 1.0 \\
\hline Dual antiplatelet therapy, n (\%) & $9(37.5)$ & $11(50)$ & $3(25)$ & 0.39 & 0.71 \\
\hline$\beta$-blockers, n (\%) & $19(79.2)$ & $17(77.3)$ & $8(66.7)$ & 1.0 & 0.44 \\
\hline Calcium channel inhibitors, n (\%) & $5(20.8)$ & $9(40.9)$ & $3(25)$ & 0.14 & 1.0 \\
\hline Nitrates, n (\%) & $7(29.2)$ & 9 (40.9) & $3(25.0)$ & 0.40 & 1.0 \\
\hline Ranolazine, n (\%) & $19(79.2)$ & $15(68.2)$ & $9(75)$ & 0.40 & 1.0 \\
\hline Ivabradine, n (\%) & $4(16.7)$ & $4(18.2)$ & $1(8.3)$ & 1.0 & 0.65 \\
\hline Number of anti-ischemic drugs, median (IQR) & $3(3-4)$ & $3(2-3)$ & $3(2-3)$ & 0.24 & 0.14 \\
\hline
\end{tabular}

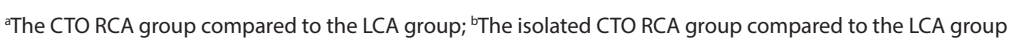

Abbreviations: CTO, chronic total occlusion; IQR, interquartile range; LCA, left coronary artery; RCA, right coronary artery; SD, standard deviation 


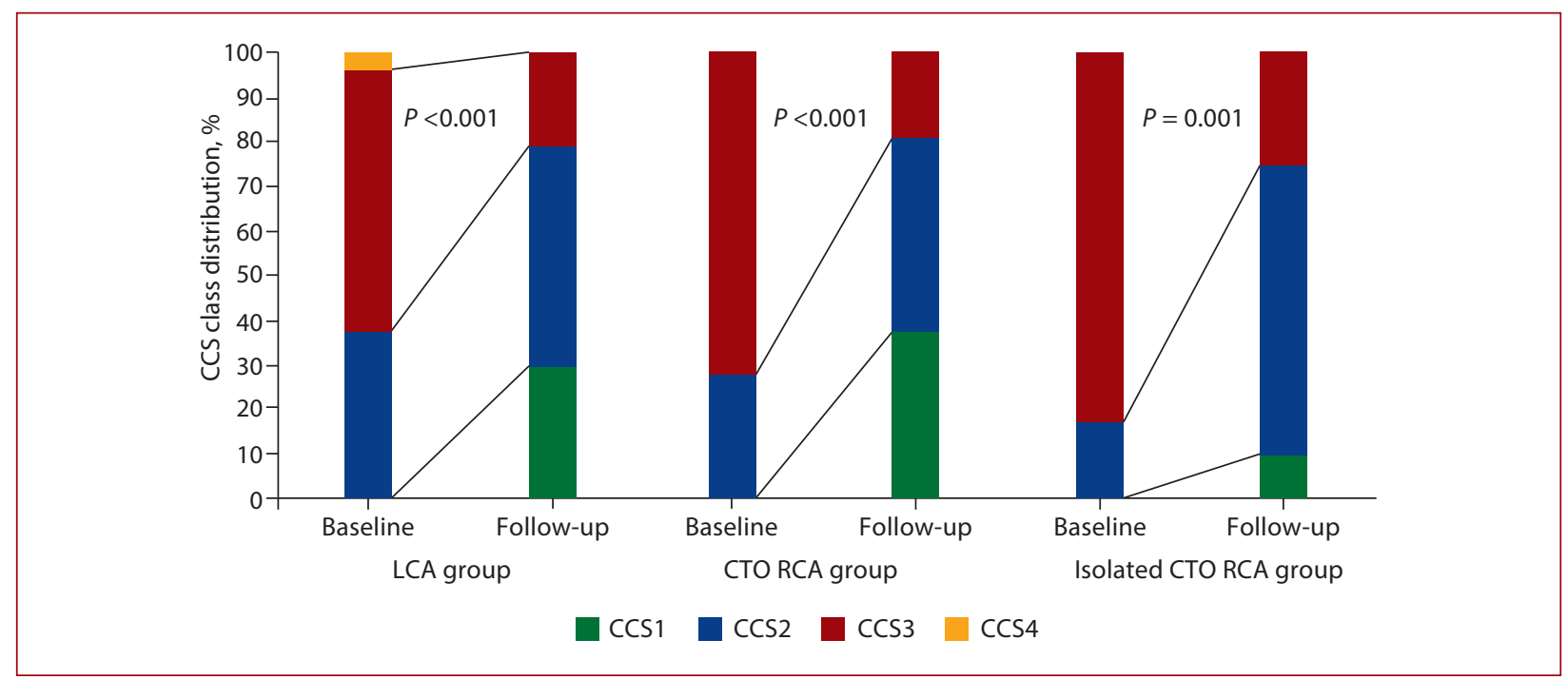

Figure 1. The Canadian Cardiovascular Society (CCS) angina score class distribution at baseline and follow-up in LCA, CTO RCA, and isolated CTO RCA group

Abbreviations: CTO, chronic total occlusion; LCA, left coronary artery; RCA, right coronary artery

al, and/or anteroseptal LVW ischemia (the LCA group). Baseline characteristics of both groups are presented in Table 1. The percutaneous coronary intervention of CTO RCA was previously attempted in 16 (72.7\%) patients, for the remaining patients, optimal medical therapy was recommended by the heart team. In CTO RCA patients, SPECT or stress CMR imaging showed predominant inferior and inferoseptal LVW ischemia while LCA patients had more diffuse ischemic areas with predominant involvement of the anterior and lateral LVW. Twelve patients in the CTO RCA group had single vessel disease and thus isolated inferior and inferoseptal LVW ischemia. All patients in the CTO RCA group had angiographically visible collateral vessels from LAD and/or LCX to the distal RCA. All patients were receiving optimal medical therapy, which did not significantly differ between the two groups $(P=0.24)$ (Table 1). Five patients in each group were not receiving beta blockers due to bradycardia or intolerance. All but 2 patients in the LCA and 3 in CTO RCA groups were receiving at least one second-line anti-ischemic drug.

\section{CSR implantation and follow-up evaluation}

CSR implantation was successful in all patients. Coronary sinus venography was performed routinely at the beginning of all procedures. CSR was implanted 1.5 to $3 \mathrm{~cm}$ distally from the ostium of the coronary sinus with caution not to obstruct any major coronary sinus tributaries. Although the follow-up time varied, there was no intergroup difference in mean (standard deviation [SD]) follow-up time - 12.7 (3.3) months in the CTO RCA group vs 13.6 (6.3) months in the LCA group $(P=0.53)$. There were 2 venous access-related complications. One patient died in the CTO RCA group due to an acute myocardial infarction 7 months following CSR implantation. One LCA patient underwent additional percutaneous coronary revascularization due to a de-novo lesion of the LCX, not previously registered during the baseline coronary angiography. Additional coronary angiography due to persistent angina was performed in $3(12.5 \%)$ LCA and 2 (9.1\%) CTO RCA patients. These were performed by attending physicians who were unaware of the study protocol or previous CSR implantation, and did not result in additional percutaneous interventions.

\section{Angina severity and quality of life assessment}

The CTO RCA and LCA groups did not differ in baseline CCS class distribution $(P=0.69)$ while analysis of SAQ domains at baseline demonstrated a trend towards worse QoL in the CTO RCA group $(P=0.056)$.

At follow-up, the CCS class improved significantly in both groups (Figure 1). In CTO RCA and in LCA groups, the mean (SD) CCS class improved from $2.73(0.46)$ to $1.82(0.73)(P<0.001)$ and from $2.67(0.57)$ to $1.92(0.72)$ $(P<0.001)$, respectively. There was no intergroup difference in CCS class improvement $(P=0.35)$. Improvement for at least one CCS class was noted in 17 (77.2\%) CTO RCA and $17(70.2 \%)$ LCA patients $(P=0.62)$. While all 5 SAQ domains improved in all groups (Figure 2), physical limitations and QoL improved significantly more in the CTO RCA group ( $P=0.001$ for both domains).

A subgroup of 12 patients with isolated CTO RCA mean (SD) CCS class improved from $2.83(0.39)$ to $2.17(0.58)$ $(P=0.001)$, which was comparable to CCS score improvement in the LCA group $(P=0.65)$. Improvement for at least one CCS class was noted in $8(66.7 \%)$ patients. Analysis of SAQ demonstrated improvement in all domains, but physical limitations and QoL improved more than in LCA patients ( $P=0.01$ and 0.03 , respectively). 


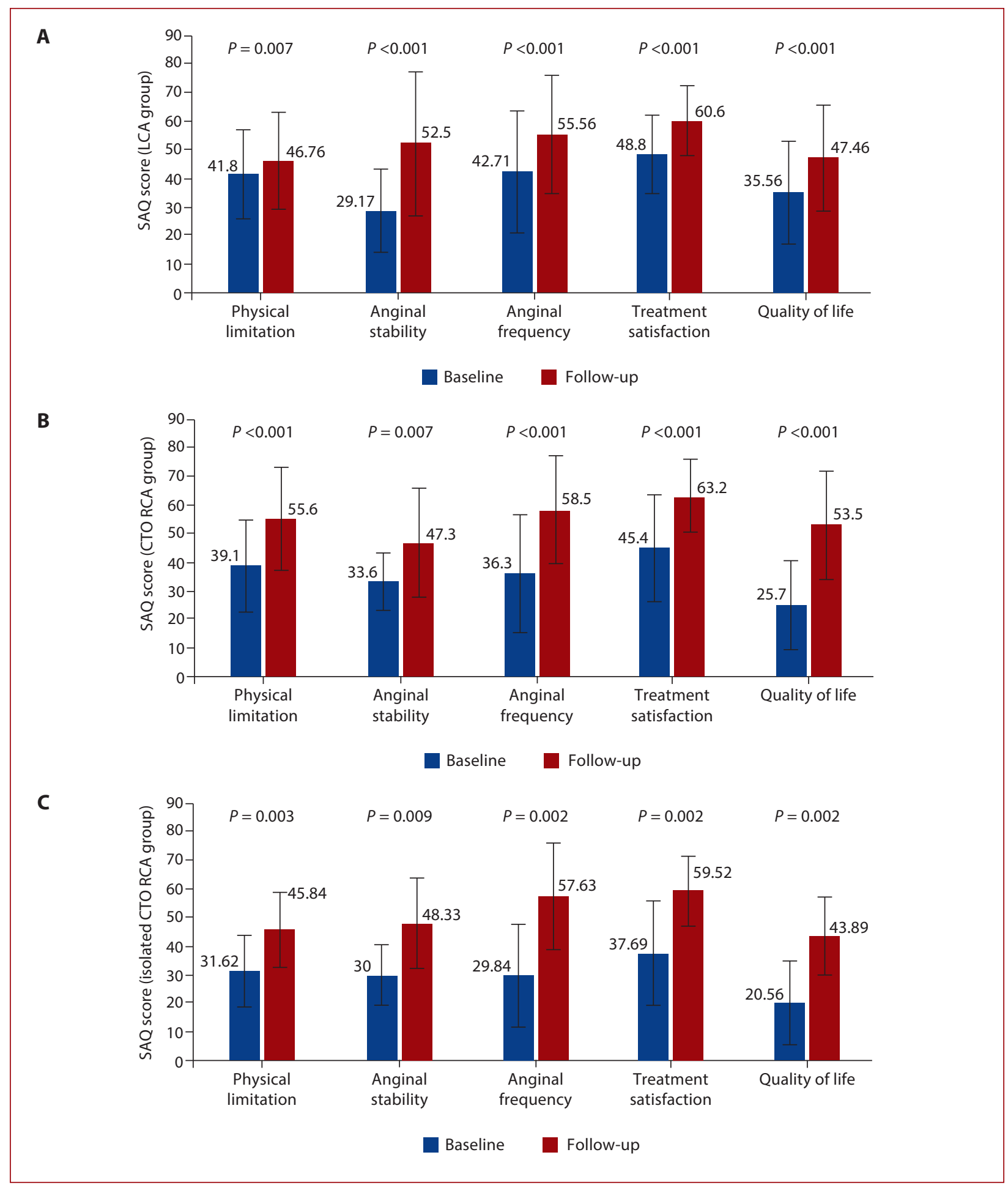

Figure 2. SAQ domains scores at baseline and follow-up in LCA (A), CTO RCA (B), and isolated CTO RCA group (C). Scores are presented as mean and standard deviation

Abbreviations: SAQ, Seattle Angina Questionnaire; other — see Figure 1

\section{Stress cardiac magnetic resonance imaging}

Ten patients in the CTO RCA group (all male, mean [SD] age 72 [7] years) completed baseline and follow-up CMR imaging protocol (Table 2). Baseline CMR showed inducible ischemia in $51 \%$ of all LV myocardial segments. Inducible ischemia was demonstrated in 43 of 50 (86\%) or a median
(IQR) of 4.5 (3.75-5) inferior and inferoseptal myocardial segments.

CMR imaging 4 months after CSR implantation demonstrated improvement in global LV myocardial perfusion as the overall number of segments with inducible ischemia decreased from $82(51 \%)$ to $70(43 \%)(P=0.02)$ 
Table 2. Stress CMR analysis in a subgroup of 10 patients with CTO RCA

\begin{tabular}{|c|c|c|c|}
\hline Left ventricular function & Baseline & 4-months & $P$-value \\
\hline Left ventricular ejection fraction, \%, mean (SD) & $63.2(17.2)$ & $65.6(11.9)$ & 0.44 \\
\hline Left ventricular end-diastolic volume, ml, mean (SD) & $130.5(33.6)$ & $133.5(45.6)$ & 0.87 \\
\hline Left ventricular end-systolic volume, ml, mean (SD) & $51.4(27.7)$ & $49.4(31.1)$ & 0.84 \\
\hline \multicolumn{4}{|l|}{ Qualitative analysis } \\
\hline Global ischemic segments, $\mathrm{n}(\%)$ & $82 / 160(51)$ & $70 / 160(43)$ & 0.02 \\
\hline Global transmurality index, median (IQR) & $1(0-1)$ & $0(0-1)$ & $<0.01$ \\
\hline Ischemic burden, $\mathrm{g}$, mean (SD) & $14.6(6.5)$ & $11.2(7.2)$ & 0.14 \\
\hline Ischemic burden, \%, mean (SD) & $20.2(7)$ & $16(10.2)$ & 0.28 \\
\hline Late Gadolinium Enhancement, \%, median (IQR) & $7(3.25-8)$ & $7(3.25-8)$ & 0.50 \\
\hline RCA ischemic segments, $\mathrm{n}(\%)$ & $43 / 50(86)$ & $39 / 50(78)$ & 0.29 \\
\hline RCA segments transmurality index, median (IQR) & $1(1-2)$ & $1(0-1)$ & 0.03 \\
\hline \multicolumn{4}{|l|}{ Semiquantitative analysis } \\
\hline Global MPRI, mean (SD) & $1.04(0.43)$ & $1.17(0.44)$ & $<0.01$ \\
\hline Global MPRI of ischemic segments, mean (SD) & $0.91(0.40)$ & $1.05(0.37)$ & 0.01 \\
\hline MPRI of all RCA segments, mean (SD) & $0.90(0.44)$ & $1.06(0.40)$ & 0.07 \\
\hline MPRI of ischemic RCA segments, mean (SD) & $0.87(0.43)$ & $1.03(0.42)$ & 0.03 \\
\hline
\end{tabular}

Ischemic segments refer to myocardial segments with inducible ischemia at baseline CMR using the AHA 16-segments LV model. Inferior and inferoseptal segments correspond to segments 3, 4, 9, 10, 15 of the AHA LV model

Abbreviations: AHA, American Heart Association; CMR, cardiac magnetic resonance; LV, left ventricular; MPRI, myocardial perfusion reserve index; other — see Table 1

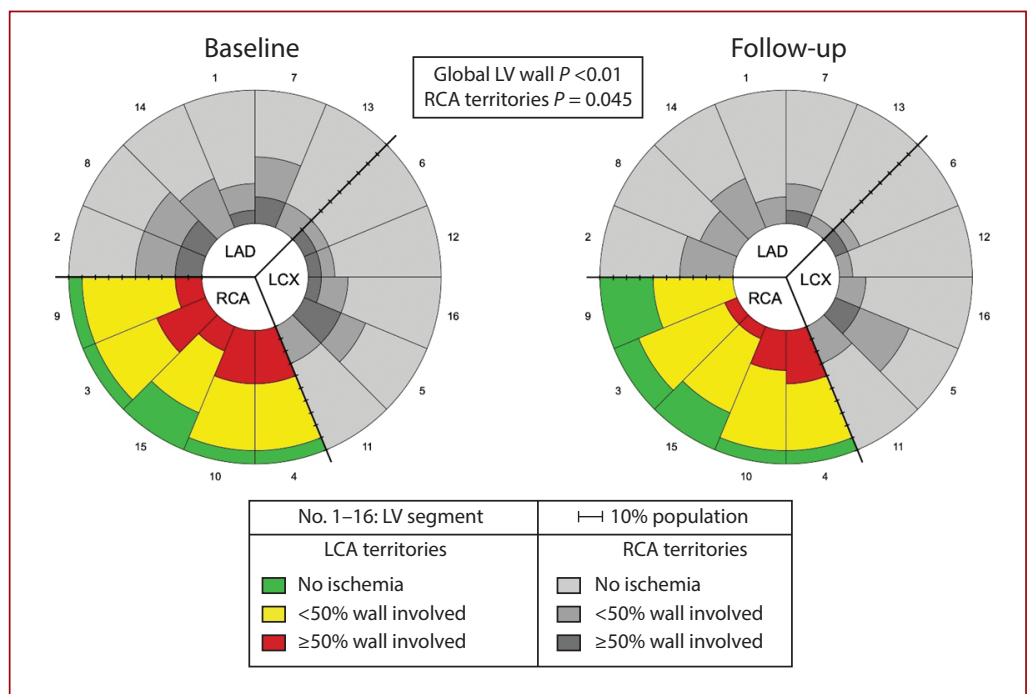

Figure 3. Qualitative analysis of myocardial ischemia by stress CMR before and after CSR implantation in 10 patients with CTO RCA related ischemia. Each sector represents a single myocardial segment of the 16-segment AHA LV model, with colored sectors representing segments $3,4,9,10$, and 15 of the inferior and inferoseptal LV wall and greyscale sectors representing remaining LV segments. Each myocardial segment is further divided as a stacked bar chart, showing the relative proportion of patients in which either absent ischemia (green or light grey), partial ischemia with $<50 \%$ wall thickness (yellow or mid-grey), or transmural ischemia with $\geq 50 \%$ of the wall was involved (red or dark grey). The median transmurality index improved significantly both globally and also in inferior and/or inferoseptal segments $(P<0.01$ and $P=0.045$, respectively)

Abbreviations: AHA, American Heart Association; CMR, cardiac magnetic resonance; CSR, coronary sinus reducer; LAD, left anterior descending; LCX, left circumflex; LV, left ventricular; other — see Figure 1

(Table 2 and Figure 3). The reduction of segments with inducible ischemia observed in the inferior and inferoseptal LVW was not significant (43 vs. 39 segments, $P=0.29$ ). There was, however, a significant improvement in both the transmurality index and the MPRI. The transmurality index decreased from a baseline median (IQR) of 1 (1-2) to a median of $1(\mathrm{IQR}, 0-1)$ at follow-up $(P=0.03)$. In segments with inducible ischemia mean (SD), the MPRI improved from $0.87(0.43)$ at baseline to $1.03(0.42)$ at follow-up $(P=0.03)$.

\section{DISCUSSION}

The results of our study are the first to show that CSR implantation in RA patients with CTO RCA and predominant inferior and inferoseptal LVW ischemia improves angina symptoms and QoL. The extent of symptomatic improvement is comparable to that observed in patients with obstructive CAD and predominant anterior, lateral, and/or anteroseptal LVW ischemia.

Before CSR implantation, patients were receiving optimized medical therapy that was in line with other CSR 
studies $[3,15]$. Improvement of angina severity in at least one CCS class following CSR implantation was observed in $77 \%$ of CTO RCA patients, which is comparable to a $70 \%$ improvement in the LCA group. A similar degree of improvement was reported in previous CSR studies, including the only randomized, sham-controlled COSIRA study where at least one CCS class reduction was noted in $71 \%$ of treated patients $[2,3,15]$. The observed reduction in the CCS class was reflected in improvement across all SAQ domains including QoL. When compared to the LCA group, patients with isolated CTO RCA had lower baseline QoL and treatment satisfaction. More pronounced symptoms at baseline might have influenced symptomatic improvement at follow-up. While CSR effects on non-dominant ischemia could partially explain the subjective improvements in the CTO RCA group, this phenomenon could not be the cause of the improvement observed in patients with isolated CTO RCA and thus isolated inferior and inferoseptal LVW ischemia. However, due to the small number of patients with isolated CTO RCA, the evidence of improvement in this subgroup should be interpreted with caution.

Patients with predominant inferior and inferoseptal LVW ischemia were traditionally excluded from CSR studies [2]. Furthermore, only one study evaluated CSR efficacy in CTO patients, showing even greater symptomatic improvement compared to patients with non-occlusive CAD [16]. In line with our results, $70 \%$ of patients with CTO RCA improved in at least one CCS class. However, those findings cannot be directly translated to patients with predominant inferior and inferoseptal LVW ischemia, as only $19 \%$ of CTO patients in the latter study presented with a single vessel disease. Due to the multifactorial etiology of myocardial ischemia, which can be present in the absence of overt coronary artery disease (e.g. in microvascular dysfunction), and possible mismatch between coronary lesions and ischemia location, we performed objective ischemia imaging before inclusion [17]. All our CTO RCA patients had SPECT or stress CMR confirmed predominant inferior and/or inferoseptal LVW ischemia. Twelve (55\%) patients had single vessel disease and thus isolated ischemia of these segments.

The observed improvement in angina severity and QoL was further complemented by indirect signs of improved myocardial perfusion as assessed by stress CMR. Imaging evidence of CSR efficacy is limited. A recent study in CSR recipients with obstructive $C A D$ and predominant anterior, lateral, and/or anteroseptal LVW ischemia demonstrated improvement in myocardial perfusion, assessed by both standard visual analysis of the number of ischemic segments and novel parameters such as the transmurality index and the MPRI [9]. In our study, the overall number of ischemic segments following CSR implantation was reduced, but we were unable to demonstrate a significant reduction of these segments in the inferior and inferoseptal LVW. However, a significant reduction in the transmurality index and increase in the MPRI in inferior and inferoseptal LVW segments was noted. Although our CMR sub-study should be regarded as exploratory due to the small number of patients and the use of novel CMR parameters, the presented results provide additional objective evidence on the possible role of CSR implantation in RA patients with CTO RCA related ischemia.

The rationale for excluding these patients from the previous studies is the cardiac venous anatomy. The inferior and inferoseptal LVW usually drains to the proximal coronary sinus and is therefore not directly amenable to CSR treatment because the device is implanted more distally. CTOs differ from non-occlusive lesions by the collateral network providing supplementary perfusion to its ischemic territory. Several collateral patterns have been described in CTO RCA patients, originating mainly from LAD and LCX [8]. All patients in our CTO RCA group had had decent collateral circulation (majority at least Rentrop grade 2). Due to the venous anatomy, there is probably no direct local effect of the increased coronary sinus pressure on the ischemia of the inferior and/or inferoseptal LVW. However, a CSR-induced increase of backward pressure in the non-ischemic region may augment coronary collateral flow and thus improve perfusion of the ischemic region exposed to lower tissue perfusion pressure $[10,18]$. As already hypothesized [16], this mechanism might be responsible for the subjective and objective improvement of the CTO RCA group observed in our study.

\section{Study limitations}

We acknowledge certain limitations of our study. The study group was relatively small and thus the conclusions should be interpreted with caution. The main limitation is the observational nature of the study with the lack of proper blinding and a sham control group. Physicians evaluating CCS scores were not blinded to the procedure, leading to potential bias. However, SAQ scores, which account for multiple aspects of angina, were evaluated by the patients themselves and correlated with the improvement measured by the CCS score. Patients with CTO RCA were compared to patients with obstructive, albeit more heterogeneous lesions of the left coronary system, which could have led to potential bias. We acknowledge that the placebo effect may have influenced the outcome in some of the patients. Patients in the sham control group of the COSIRA study demonstrated a $40 \%$ improvement for at least one CCS class, which is comparable to the placebo effect observed in the previous studies enrolling angina pectoris patients $[2,19]$. The magnitude of CCS improvement observed in our study was significantly greater than what would be expected solely by the placebo effect and was similar to the improvement seen in the CSR group of the COSIRA study. Furthermore, to minimize the placebo effect, CRS efficacy in CTO RCA patients was compared to a control group of LCA patients who also received the 
same device therapy. Lastly, although only exploratory, we provide objective evidence of improved myocardial perfusion by stress CMR imaging.

\section{CONCLUSIONS}

Coronary sinus reducer could be beneficial in patients with CTO RCA-related inferior and/or inferoseptal LVW ischemia with the observed reduction in angina severity and improved QoL. The alleviation of angina and QoL improvement were comparable to that observed in patients with obstructive CAD and predominant anterior and lateral LVW ischemia. Further larger prospective studies are warranted to address the potential clinical benefit of CSR in this group of patients.

\section{Article information}

Conflict of interest: SB is the medical director of Neovasc. FG is a consultant for Neovasc. All other authors have no conflicts of interest to declare.

Open access: This article is available in open access under Creative Common Attribution-Non-Commercial-No Derivatives 4.0 International (CC BY-NC-ND 4.0) license, allowing to download articles and share them with others as long as they credit the authors and the publisher, but without permission to change them in any way or use them commercially. For commercial use, please contact the journal office at kardiologiapolska@ptkardio.pl.

\section{REFERENCES}

1. Parikh $P, B$ hatt $P$, Shah $D$, et al. First-in-Human use of coronary sinus reducer in patients with refractory angina. J Am Coll Cardiol. 2018; 72(24) 3227-3228, doi: 10.1016/j.jacc.2018.09.061, indexed in Pubmed: 30545460.

2. Verheye S, Jolicœur EM, Behan MW, et al. Efficacy of a device to narrow the coronary sinus in refractory angina. N Engl J Med. 2015; 372(6): 519-527, doi: 10.1056/NEJMoa1402556, indexed in Pubmed: 25651246.

3. Giannini F, Baldetti L, Konigstein M, et al. Safety and efficacy of the reducer: a multi-center clinical registry — REDUCE study. Int J Cardiol. 2018; 269: 40-44, doi: 10.1016/j.ijcard.2018.06.116, indexed in Pubmed: 29983248.

4. Giannini F, Baldetti L, lelasi A, et al. First experience with the coronary sinus reducer system for the management of refractory angina in patients without obstructive coronary artery disease. JACC CardiovasC Interv. 2017; 10(18): 1901-1903, doi: 10.1016/j.jcin.2017.06.062, indexed in Pubmed: 28935084.

5. ZimochW, KuliczkowskiW, Reczuch K. Coronary sinus reducer implantation in patients with refractory angina: first experience in Poland. Kardiol Pol. 2021; 79(4): 471-472, doi: 10.33963/KP.15866, indexed in Pubmed: 33687874.

6. Ponticelli F, Khokhar AA, Leenders G, et al. Safety and efficacy of coronary sinus narrowing in chronic refractory angina: Insights from the RESOURCE study. Int J Cardiol. 2021; 337: 29-37, doi: 10.1016/j.ijcard.2021.05.034, indexed in Pubmed: 34029618.
7. Konigstein M, Giannini F, Banai S. The Reducer device in patients with angina pectoris: mechanisms, indications, and perspectives. Eur Heart J. 2018; 39(11): 925-933, doi: 10.1093/eurheartj/ehx486, indexed in Pubmed: 29020417.

8. McEntegart MB, Badar AA, Ahmad FA, et al. The collateral circulation of coronary chronic total occlusions. Eurolntervention. 2016; 11(14): e1596-e1603, doi: 10.4244/EIJV11/14A310, indexed in Pubmed:27056120.

9. Giannini F, Palmisano A, Baldetti L, et al. Patterns of regional myocardial perfusion following coronary sinus reducer implantation: insights by stress cardiac magnetic resonance. Circ Cardiovasc Imaging. 2019; 12(9): e009148, doi: 10.1161/CIRCIMAGING.119.009148, indexed in Pubmed: 31451003.

10. Ido A, Hasebe $\mathrm{N}$, Matsuhashi $\mathrm{H}$, et al. Coronary sinus occlusion enhances coronary collateral flow and reduces subendocardial ischemia. Am J Physiol Heart Circ Physiol. 2001; 280(3): H1361-H1367, doi: 10.1152/ajpheart.2001.280.3.H1361, indexed in Pubmed: 11179085.

11. Sato M, Saito T, Mitsugi M, et al. Effects of cardiac contraction and coronary sinus pressure elevation on collateral circulation. Am J Physiol. 1996; 271(4 Pt 2): H1433-H1440, doi: 10.1152/ajpheart.1996.271.4.H1433, indexed in Pubmed: 8897937.

12. Giannini F, Tzanis G, Ponticelli F, et al. Technical aspects in coronary sinus Reducer implantation. Eurolntervention. 2020; 15(14): 1269-1277, doi: 10.4244/EIJ-D-18-01180, indexed in Pubmed: 30967360.

13. Hussain ST, Paul M, Plein S, et al. Design and rationale of the MR-INFORM study: stress perfusion cardiovascular magnetic resonance imaging to guide the management of patients with stable coronary artery disease. J Cardiovasc Magn Reson. 2012; 14: 65, doi: 10.1186/1532-429X-14-65, indexed in Pubmed: 22992411.

14. Schulz-Menger J, Bluemke DA, Bremerich J, et al. Standardized image interpretation and post processing in cardiovascular magnetic resonance: Society for Cardiovascular Magnetic Resonance (SCMR) board of trustees task force on standardized post processing. J Cardiovasc Magn Reson. 2013; 15(1): 35, doi: 10.1186/1532-429X-15-35, indexed in Pubmed: 23634753

15. Konigstein M, Bazan S, Revivo M, et al. Coronary Sinus Reducer implantation improves symptoms, ischaemia and physical capacity in patients with refractory angina unsuitable for myocardial revascularisation: a single-centre experience. Eurolntervention. 2018; 14(4): e452-e458, doi: 10.4244/EIJ-D-18-00102, indexed in Pubmed: 29688178.

16. Zivelonghi $C$, Verheye $S$, Timmers $L$, et al. Efficacy of coronary sinus reducer in patients with non-revascularized chronic total occlusions. Am J Cardiol. 2020; 126: 1-7, doi: 10.1016/j.amjcard.2020.03.042, indexed in Pubmed: 32345474.

17. Marzilli M, Crea F, Morrone D, et al. Myocardial ischemia: from disease to syndrome. Int J Cardiol. 2020; 314:32-35, doi: 10.1016/j.jijcard.2020.04.074, indexed in Pubmed: 32348810.

18. Stoller M, Traupe T, Khattab AA, et al. Effects of coronary sinus occlusion on myocardial ischaemia in humans: role of coronary collateral function. Heart. 2013; 99(8): 548-555, doi: 10.1136/heartjnl-2012-303305, indexed in Pubmed: 23343686.

19. Leon MB, Kornowski R, Downey WE, et al. A blinded, randomized, placebo-controlled trial of percutaneous laser myocardial revascularization to improve angina symptoms in patients with severe coronary disease. J Am Coll Cardiol. 2005; 46(10): 1812-1819, doi: 10.1016/j.jacc.2005.06.079, indexed in Pubmed: 16286164 